\title{
QT interval dispersion: a non-invasive marker of susceptibility to arrhythmia in patients with sustained ventricular arrhythmias?
}

\author{
M Pye, A C Quinn, S M Cobbe
}

\begin{abstract}
Objective-To assess QT interval dispersion on the surface electrocardiogram in patients with sustained ventricular arrhythmias.

Design-A retrospective and prospective blinded controlled study of patients referred for investigation of ventricular arrhythmias at a tertiary cardiac centre. Patients and methods-89 consecutive patients with sustained ventricular arrhythmias due to chronic ischaemic heart disease, cardiomyopathy, or ventricular tachycardia (VT) in a normal heart. 32 patients did not meet the inclusion criteria; therefore 57 patients were compared with a control group of 40 patients with myocardial disease but no history of arrhythmias and 12 normal controls with no myocardial disease. Standard 12 lead electrocardiograms were enlarged, the QT intervals for each lead measured, and QT dispersion calculated.
\end{abstract}

Results-There was a significantly greater mean QT dispersion $(77 \mathrm{~ms})$ in patients with sustained ventricular arrhythmias compared with the control group (38 ms, p < 0.01). This held for all groups; after myocardial infarction VT (82 (22) ms $v$ control 38 (10) ms; p < 0.01), dilated cardiomyopathy VT (76 (18) ms $v$ control 40 (11) ms, p $<0.01$ ), and normal heart VT (65 (7) ms $v$ control 32 (8), p < 0.05). There was also a greater QT dispersion in patients with impaired left ventricular function and VT, with a correlation between left ventricular function and QT dispersion in patients with VT $(r=0.56, p<0.01)$.

Conclusion-QT interval dispersion may be a further non-invasive marker of susceptibility to ventricular arrhythmias.

(Br Heart f 1994;71:511-514)

Department of Cardiology, Royal Infirmary, Glasgow, Scotland

M Pye

A C Quin

A C Quinn

Correspondence to:

Dr M Pye, Department of Dr M Pye, Department of
Cardiology, St Georges Lospital, Blackshaw R.

Accepted for publication 6 December 1993
A long QT interval has been associated with a risk of arrhythmias in the hereditary long QT syndromes ${ }^{1}$ and seems to identify high risk survivors of acute myocardial infarction. ${ }^{2-4} \mathrm{~A}$ single derived value of the QT interval from the electrocardiogram suggests that this is a representative value for the whole of cardiac repolarisation. It is well known that there is dispersion of repolarisation across the ventricular myocardium in the normal heart. ${ }^{5}$ Such temporal dispersion of recovery is also widely accepted as an important factor in the pathogenesis of ventricular arrhythmias, as it increases the vulnerability to ventricular re-entry and sustained tachycardias. ${ }^{67}$ The adjacent difference in repolarisation of refractoriness may be the crucial factor allowing re-entry to occur. ${ }^{8}$ Previous work has shown that the QT interval varies from lead to lead of the surface electrocardiogram creating QT dispersion. ${ }^{9}$ This variation could either be due to technical factors or represent true regional differences in repolarisation across the ventricular myocardium with recent evidence strongly supporting the true regional differences. ${ }^{1011}$ Therefore QT interval dispersion may be a marker of susceptibility to ventricular arrhythmias as has been shown in hereditary long QT syndromes. ${ }^{12} 13$

The aims of the study were to measure QT interval dispersion from the surface electrocardiograms of patients with a history of sustained ventricular tachycardia (VT) or ventricular fibrillation (VF) due to a variety of aetiologies and compare them to a control group with diseased myocardium but no history of arrhythmias.

\section{Patients and methods}

The simultaneous 12 lead electrocardiograms from all patients with a documented history of sustained VT or VF referred to an electrophysiology centre were analysed unless exclusion criteria were met.

\section{EXCLUSION CRITERIA}

Patients were excluded if at the time of the electrocardiographic recording there was any evidence of acute ischaemia or myocardial infarction in the preceding eight weeks. The electrocardiogram was recorded on a machine capable of simultaneous 12 lead acquisition. Patients had to be in sinus rhythm with no bundle branch block. They were not allowed to be taking any antiarrhythmic drugs or drugs known to affect the QT interval and the serum potassium concentration had to be $>3.9 \mathrm{mmol} / 1$.

Of 89 patients screened 32 were excluded because of unmeasurable $T$ waves, atrial fibrillation, bundle branch block, or nonavailability of electrocardiograms with simultaneous 12 lead recording. This left 57 patients (52 with sustained monomorphic VT $>2 \mathrm{~min}$ and five with VF). Included were 40 with a history of remote myocardial infarction, nine with dilated cardiomyopathy, and eight with a normal heart (normal coronary 
Table 1 Comparison of clinical data from patients with VT or VF and controls

\begin{tabular}{lll}
\hline After myocardial infarction: & $\begin{array}{l}\text { VT or VF } \\
\text { group }\end{array}$ & $\begin{array}{l}\text { Control } \\
\text { group }\end{array}$ \\
\hline No & 40 & 30 \\
Mean age (yr) & 64 & 63 \\
Sex (M) & 32 & 23 \\
Mean LVEF (\%) & 38 & 41 \\
Heart rate (beats/min) & 75 & 73 \\
Dilated cardiomyopathy: & & \\
No & 9 & 10 \\
Mean age (yr) & 61 & 60 \\
Sex (M) & 5 & 5 \\
Mean LVEF (\%) & 37 & 40 \\
Heart rate (beats/min) & 73 & 72 \\
Normal heart: & 8 & 12 \\
No & 51 & 49 \\
Mean age (yr) & 4 & 5 \\
Sex (M) & 59 & 61 \\
Mean LVEF (\%) & 70 & 67 \\
Heart rate (beats/min) & & \\
\hline
\end{tabular}

LVEF, left ventricular ejection fraction.

angiogram, normal echocardiogram, including right ventricular views, and no evidence of a long QTc ( $<440 \mathrm{~ms})$ ).

The control group consisted of 52 patients with no history of arrhythmia or syncope. Thirty control patients had a diagnosis of previous myocardial infarction, 10 had idiopathic dilated cardiomyopathy, and 12 were normal volunteers with no evidence of cardiac disease on clinical, electrocardiographic, or echocardiographic grounds. Table 1 shows the clinical data for the study and control groups.

\section{QT ANALYSIS}

Standard electrocardiograms with simultaneous 12 lead acquisition were recorded at 25 $\mathrm{mm} / \mathrm{s}$. They were enlarged on the same photocopier by a factor of three. We had found in a pilot study that recording the electrocardiogram at $50 \mathrm{~mm} / \mathrm{s}$ did not improve the accuracy of the QT measurement as it resulted in obscuring the end of the $T$ wave. A blinded observer measured the QT intervals manually with calipers from the onset of the QRS to the end of the $T$ wave defined as the return to TP baseline. When $U$ waves were present the QT interval was measured to the nadir of the curve between the $T$ and $U$ waves. Three consecutive cycles were measured in each of the standard 12 leads and from the three values a mean QT was calculated. When the end of the $T$ wave could not be identified the lead was not included. A minimum of seven leads, at least three precordial, was required for QT dispersion to be calculated.

The QT dispersion was defined as the difference between the maximum and minimum QT interval occurring in any of the 12 electro-

Table $2 Q T$ dispersion in patients with VT of varying aetiology and in respective control groups

\begin{tabular}{lll}
\hline Group & $n$ & $\begin{array}{l}\text { Mean (SD) } Q T \\
\text { dispersion (ms) }\end{array}$ \\
\hline After MI VT & 40 & $82(22)$ \\
After MI control & 30 & $38(10)$ \\
DCM VT & 9 & $76(18)$ \\
DCM control & 10 & $40(11)$ \\
Normal heart VT & 8 & $65(7)$ \\
Normal heart control & 12 & $32(8)$ \\
\hline
\end{tabular}

DCM, dilated cardiomyopathy; MI, myocardial infarction. cardiographic leads. The maximum adjacent QT dispersion was defined as the maximum difference between two adjacent precordial leads as it has been suggested that it is the adjacent dispersion in recovery rather than total dispersion that is important in arrhythmogenesis. ${ }^{8}$

\section{LEFT VENTRICULAR FUNCTION}

This was assessed by cross sectional echocardiography (with modified Simpson's formula) or resting gated technetium-99m radionuclide scanning with the normal range for left ventricular ejection fraction $>55 \%$. The ejection fraction data obtained from the two different methods were converted to a comparable contrast angiographic ejection fraction with previously verified formulae. ${ }^{14} 15$

\section{REPRODUCIBILITY}

We performed a study on the variability of QT measurements. Ten electrocardiograms were coded and duplicated and measured blind twice by two observers. The percentage differences in QT measurements for the same electrocardiogram ranged from $2 \%-4 \%$ for within observer variability and 3\%-5\% for between observer variability.

STATISTICS

Student's unpaired $t$ test was used to compare the group means.

\section{Results}

Table 1 shows the clinical data for the study and control groups. They were well matched for age, sex, left ventricular function, and heart rate at the time of the electrocardiographic recording. As a heterogeneous group patients with VT had a significantly greater mean QT interval dispersion $(77 \mathrm{~ms})$ than patients without VT $(38 \mathrm{~ms}, \mathrm{p}<0.01)$. Similarly, the maximum adjacent QT dispersion was significantly greater in the VT group (43 ms) than in the control group without VT (31 ms, p < 0.05).

Table 2 shows the results for the mean (SD) QT dispersion on the basis of aetiological subgroup. Patients with VT from all aetiologies had a significantly greater QT dispersion than the relevant control group (for the after myocardial infarction VT group 82 (22) ms $v$ $38(10) \mathrm{ms}, \mathrm{p}<0.01$; for the dilated cardiomyopathy VT group 76 (18) ms $v 40$ (11) ms, $\mathrm{p}<0.01$; for the normal heart VT group 65 (7) ms $v 32$ (8) ms, p < 0.05).

Table 3 shows the results for QT dispersion on the basis of left ventricular function. Patients with VT and impaired left ventricular

Table $3 Q T$ dispersion on the basis of left ventricular function in patients with VT and in a control group without VT

\begin{tabular}{llll}
\hline Group & Ejection fraction (\%) & $n$ & $\begin{array}{l}\text { Mean (SD) } \\
\text { QTdispersion (ms) }\end{array}$ \\
\hline VT & $<40$ & 39 & $84(21)$ \\
VT & $>40$ & 18 & $58(17)$ \\
Control & $<40$ & 22 & $41(15)$ \\
Control & $>40$ & 30 & $35(14)$ \\
\hline
\end{tabular}


Figure Correlation between $Q T$ dispersion and left ventricular (LV) ejection fraction in patients with ventricular tachycardia.

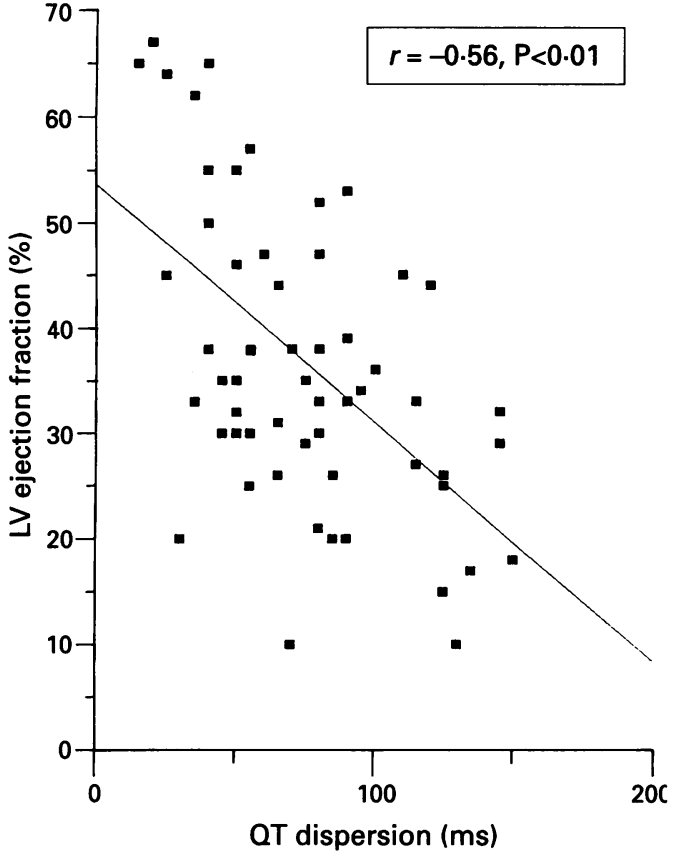

function (ejection fraction $<40 \%$ ) had a significantly greater QT dispersion $(84 \mathrm{~ms})$ than patients with VT and good left ventricular function $(58 \mathrm{~ms}, \mathrm{p}<0.01)$. There seemed to be no significant independent effect of left ventricular function on QT dispersion in patients without VT $(p=0 \cdot 16)$. There was a significant correlation between QT dispersion and left ventricular ejection fraction in patients with VT $(r=0.56, \mathrm{p}<0.01$, figure).

There was a non-significant trend towards a longer maximum QT interval in the VT group (mean (SD), $463(65) \mathrm{ms}$ ) than in the control group (439 (56) ms, $\mathrm{p}=0.08)$.

\section{Discussion}

These results are further evidence that QT interval dispersion may be a non-invasive marker of susceptibility to arrhythmias in patients with VT from a variety of aetiologies. This suggests that a single value for the QT interval from the surface electrocardiogram is not appropriate and far more information regarding ventricular repolarisation is available by calculating QT dispersion. There are problems in accurate measurement of the QT interval particularly in identifying the end of the $T$ wave. With careful selection of electrocardiograms, however, with respect to $T$ wave configuration, an acceptable low within observer measurement variability is reached. Other workers have also found low within and between observer variability for QT interval measurements with selection of appropriate electrocardiograms. ${ }^{5}$ In future the measurement of QT intervals and QT dispersion could be improved by computerised electrocardiographic waveform analysis with automatic QT interval measurement for each lead.

The concept that QT interval dispersion reflects dispersion of repolarisation is supported by the close correlation between change in dispersion of repolarisation, from ventricular monophasic action potential recordings, and change in QT interval dispersion produced by ventricular pacing, ${ }^{9-11}$ in patients without ventricular arrhythmias. Patients with the long QT syndrome ${ }^{12}$ and torsades de pointes ${ }^{13}$ also have increased QT interval dispersion and increased dispersion of ventricular repolarisation recorded with monophasic action potentials. ${ }^{16}{ }^{17}$ Bashir et al found an increased QTc interval dispersion (mean $97 \mathrm{~ms}$ ) in 14 patients with a history of sustained VT after a myocardial infarction compared with $57 \mathrm{~ms}$ in a control group without VT. ${ }^{18}$ The same relation did not hold for patients with only high grade ventricular extrasystole without sustained VT after a myocardial infarction. ${ }^{19}$

We found evidence of an increased dispersion of QT interval in patients with VT and impaired left ventricular function. This may be related to the phenomenon of contraction excitation feedback occurring in heart failure, ${ }^{20}$ whereby mechanical stretch or dilatation of the myocardium alters the electrophysiological properties of the myocardium. Alternatively QT dispersion could be an epiphenomenon reflecting indirectly the degree of left ventricular dysfunction rather than being a direct marker of susceptibility to arrhythmia.

Ventricular re-entry and arrhythmogenesis are known to be favoured by certain electrophysiological properties-namely, slow conduction, unidirectional conduction block, and dispersion of refractoriness, which are also affected by various autonomic factors. The degree of overlap in QT interval dispersion between patients with and without VT found in our study may not make it a useful single predictor of arrhythmogenicity. It might be more fruitful to combine the various noninvasive markers of these arrhythmogenic properties - that is, the signal averaged electrocardiogram for detection of late potentials as an indicator of slow or fragmented ventricular conduction, ${ }^{21}$ baroreflex sensitivity and heart rate variability as a measure of altered autonomic tone, ${ }^{22}{ }^{23}$ and QT interval dispersion representing dispersion of ventricular recovery. This could improve the predictive accuracy of arrhythmic events without resort to invasive electrophysiological studies with their attendant problems and cost. ${ }^{24}$

1 Schwartz PJ, Periti M, Malliani A. The long QT syndrome. Am Heart f 1975;89:378-90.

2 Schwartz PJ, Wolf S. QT interval prolongation as a predictor of sudden death in patients with myocardial infarctor of sudden death in patients with

3 Ahnve S, Helmers C, Lundman T, Rehnquist N, Sjogren A QTc intervals in acute myocardial infarction: first yea prognostic implications. Clin Cardiol 1980;3:303-8.

4 Ahnve S, Helmers C, Lundman T. Q-Tc intervals at discharge after acute myocardial infarction and long term prognosis. Acta Med Scand 1980;208:55-60.

5 Cowan JC, Yusoff $\mathrm{K}$, Moore M, et al. Importance of lead selection in QT interval measurement. $A m \mathcal{f}$ Cardio 1988;61:83-7.

Han J, Moe GK. Non uniform recovery of excitability in ventricular muscle. Circ Res 1964;14:44-50.

7 Merx W, Yoon MS, Han J. The role of local disparity in conduction and recovery time on ventricular vulnerability to fibrillation. Am Heart $\mathcal{f} 1977 ; 94: 603-12$. 
8 Restivo M, Gough WB, El-Sherif N. Ventricular arrhythmias in the subacute myocardial infarction period: high resolution activation and refractory patterns of reentrant rhythms. Circ Res 1990;66:1310-27.

9 Higham PD, Hilton CJ, Griffiths CJ, Furniss CS, Campbell RWF. Relation between QT intervals and epicardial monophasic action potentials [abstract]. Br Heart f 1992;63:123.

10 Day CP, McComb J, Campbell RWF. QT dispersion in sinus beats and ventricular extrasystoles in normal hearts. Br Heart $\tilde{f} 1992 ; 67: 39-41$.

11 Moran JM Cunningam D, Rowland E. Dispersion of monophasic action potential duration: demonstrable in monophasic action potential duration: demonstrable in humans after premature ventricular stimulation but

12 Day CP, McComb JM, Campbell RWF. QT dispersion: an indication of arrhythmia risk in patients with long QT in inds. $B r$ Heart $\mathcal{Y} 1990 ; 63: 342-4$.

13 De Ambrioggi L, Negroni MS, Monza E, Bertoni T, Schwartz PJ. Dispersion of ventricular repolarisation in the long QT syndrome. Am $\mathcal{F}$ Cardiol 1991;68:614-20.

14 McGhie I, Tweddel A, Martin W, Hutton I. Detection of coronary artery disease; a comparison of exercise thallium imaging and exercise equilibrium radionuclide ventriculography. Eur F Nucl Med 1987;13:18-23.

15 Erbel R, Schweizer P, Lambertz H, Henn G, Meyer J, Krebs W, et al. Echoventriculography-a simultaneous analysis of two dimensional echocardiography and cineventriculography. Circulation 1983;67:205-11.

16 Gavrilescu S, Luca C. Right ventricular monophasic action potentials with the long QT syndrome. $\mathrm{Br}$ Heart $\mathcal{f}$ 1978;40:1014-8.

17 Bonatti V, Rolti A, Bolti G. Recording of monophasic action potentials of right ventricle in long QT syndromes complicated by ventricular arrhythmias. Eur Heart $\mathcal{f}$ 1983;4:168-74.

18 Bashir Y, Farrell T, Camm AJ. Increased QT dispersion in survivors of acute myocardial infarction who subsequently develop ventricular arrhythmias or sudden death [abstract]. PACE 1990;13:561.

19 Dristas A, Sbarouni E, Nihoyannopoulos P, Cleland J, Oakley C. Relation of QTc interval and QTc dispersion Oo myocardial infarction [abstract]. Eur Heart $\mathcal{f}$ 1992;13: 344.

20 Pye MP, Cobbe SM. Mechanisms of ventricular arrhythmias in cardiac failure and hypertrophy. Cardiovasc Res mias in cardiac

21 Kleiger RE, Miller JP, Bigger JT Jr, Moss AJ and the Multicentre Post-Infarction Research Group. Heart rate valticentre Post-Infarction Research Group. Heart rate variability: a predictor of mortality following acute

22 Farrell TG, Bashir Y, Cripps T, Malik M, Polonieki J, Bennett D, et al. Risk stratification for arrhythmic event in postinfarction patients based on heart rate variability, ambulatory electrocardiographic variables and the signal averaged electrocardiogram. F Am Coll Cardiol 1991;18: 687-97.

23 Farrell TG, Paul V, Cripps TR, Malik M, Bennett ED, Ward D, et al. Baroreflex sensitivity and electrophysiological correlates in patients after acute myocardial infarction. Circulation 1991;83:945-52.

24 Dennis AR, Richards DA, Cody DV, Russell PA, Young AA, Ross DL, et al. Prognostic significance of ventricular tachycardia and fibrillation induced at pro tachycardia and fibrillation induced at programmed ventricular stimulation and delayed potentials on signal myocardial infarction. Circulation 1986;74:731-45. 